\title{
Desabastecimento de medicamentos na literatura científica da saúde: uma revisão narrativa
}

\author{
I ${ }^{1}$ Luisa Arueira Chaves, ${ }^{2}$ Gabriela Costa Chaves, ${ }^{3}$ Mariani Nunes Sadock Vianna, \\ ${ }^{4}$ Maria Auxiliadora Oliveira I
}

Resumo: O desabastecimento de medicamentos já é considerado um problema de saúde pública e representa um obstáculo importante para a garantia do acesso a eles e, consequentemente, do direito à saúde. Sendo assim, com o intuito de compreender melhor esse fenômeno, este artigo buscou identificar, descrever e caracterizar as publicaçóes científicas da saúde que versam sobre o tema de desabastecimento de medicamentos e identificar as lacunas de pesquisa. Para tanto, realizou-se revisão narrativa da literatura científica na base de dados PubMed. Os resultados foram selecionados de acordo com o título e resumo, e os dados foram extraídos do texto completo. Além de uma análise quantitativa, também foi realizada uma síntese qualitativa dos estudos, explicitando as principais causas, estratégias de enfrentamento, discussôes conceituais e a descrição do problema contido nas publicaçóes incluídas. Foram analisados 98 artigos, a maioria foi publicada a partir do ano de 2011, nos EUA, citando diversos medicamentos e com o foco na descrição do problema. Os resultados deste estudo sugerem a contemporaneidade do problema, o uso da literatura científica como denúncia e a falta de estudos sobre o tema em países de baixa e média renda e que se voltem a compreender suas causas.

> Palavras-chave: preparações farmacêuticas; revisão; acesso aos serviços de saúde.

\author{
1 Universidade Federal do Rio de \\ Janeiro, Campus Macaé. Macaé-RJ, \\ Brasil (luisa.arueira@gmail.com). \\ ORCID: 0000-0002-9597-3651. \\ ${ }^{2}$ Escola Nacional de Saúde \\ Pública Sergio Arouca, Fiocruz. \\ Rio de Janeiro-RJ, Brasil \\ (gabicostachaves@ensp.fiocruz.br). \\ ORCID: 0000-0002-8347-6164. \\ ${ }^{3}$ Escola Nacional de Saúde \\ Pública Sergio Arouca, Fiocruz. \\ Rio de Janeiro-RJ, Brasil \\ (msadock@id.uff.br). \\ ORCID: 0000-0002-7635-0395. \\ ${ }^{4}$ Escola Nacional de Saúde Pública \\ Sergio Arouca, Fiocruz. Rio de \\ Janeiro-RJ, Brasil (dorabermudez@ \\ gmail.com). \\ ORCID: 0000-0003-2400-536X.
}




\section{Introdução}

Em 2016, a falta do medicamento penicilina cristalina foi tema dos principais jornais brasileiros (BOM DIA BRASIL, 2016; MARTINS, 2016). Este medicamento é um dos principais tratamentos para sífilis em gestantes e neonatos e sua falta apresentou efeitos importantes no controle e tratamento dessa doença (BRASIL, 2015). Este problema, no entanto, não está limitado a um medicamento, tampouco a um grupo específico de medicamentos ou ao cenário nacional.

Ele tem transcendido fronteiras nacionais e já vem sendo debatido em arenas internacionais, sendo tema de discussão da Assembleia Mundial da Saúde (AMS) de 2016 (WHO, 2016a) e de reunióes do Conselho Executivo em 2015, 2017 e 2018 (WHO, 2015; 2017; 2018). As discussóes no âmbito da Organização Mundial da Saúde (OMS) significam, portanto, que o desabastecimento de medicamentos tornou-se um problema de amplitude mundial.

Considerando que a falta do medicamento no ponto da dispensação decorrente do desabastecimento diferencia-se da baixa disponibilidade por conta de financiamento insuficiente e/ou problemas da gestão local da assistência farmacêutica, é necessário definir o conceito de desabastecimento. O dicionário define desabastecimento como "Ato ou efeito de eliminar o fornecimento de algo"; ou, ainda, "Falta de produtos no mercado" (MICHAELIS, [s.d.]). A OMS, por outro lado, diferencia "shortage" de "stock out" e conceitua desabastecimento tanto pelo lado da demanda quanto pelo lado da oferta. Segundo esta definição, desabastecimento ("shortage"), pelo lado da oferta, seria quando a produção é insuficiente para atender à demanda da população. Convém ressaltar que só vale para produtos que já estejam aprovados e registrados. Já o desabastecimento pelo lado da demanda ("shortage”) refere-se a um aumento abrupto da demanda, resultando em indisponibilidade no ponto de dispensação (WHO, 2016b).

No Brasil, a Agência Nacional de Vigilância Sanitária (Anvisa) conceituou descontinuação temporária e definitiva de produção ou importação de medicamentos (BRASIL, 2014b). Na prática, esta definiçãoconsideraaiminência de desabastecimento a partir do potencial de descontinuação, e não a falta propriamente dita. Desta forma, pode-se dizer que não foi encontrada uma definiçẫo oficial para desabastecimento. $\mathrm{O}$ que se sabe é que quando ocorre um desabastecimento de medicamentos, tem-se 
como consequência disponibilidade baixa ou indisponibilidade total nos pontos de dispensação, podendo comprometer, portanto, o cuidado em saúde. Considerando que a disponibilidade é uma das dimensões do acesso a medicamentos (FROST; REICH, 2008), pode-se dizer que o desabastecimento de medicamentos é um de seus obstáculos, afetando diretamente este acesso.

O desabastecimento é, portanto, um dos obstáculos para o acesso a medicamentos que necessitam de novas estratégias de enfrentamento para a garantia do direito à saúde das populaçóes. Em um momento no qual se comemoram os grandes avanços da ciência e novas entidades moleculares capazes de tratar diversas patologias, chama a atenção que os medicamentos que já existem e que são consagrados na assistência à saúde estão, literalmente, sumindo do mercado (GEHRETT, 2012). Adicionalmente, para o campo do acesso a medicamentos, este problema traz a necessidade de se pensar em novas formas de análise e de atuação, uma vez que a garantia do financiamento, os sistemas de informação, a capacitaçáo de recursos humanos e os sistemas logísticos eficientes não parecem ser suficientes para o enfrentamento desta situação.

Este artigo tem por objetivo identificar, descrever e caracterizar as publicaçóes científicas da área biomédica que versam sobre o tema de desabastecimento de produtos farmacêuticos e identificar as lacunas sobre o tema na literatura científica.

\section{Método}

Este é um estudo de revisão narrativa com o tema de desabastecimento de produtos farmacêuticos. Foi escolhida a revisão narrativa como método no intuito de mapear a literatura indexada em bases científicas sobre o tema, propiciando, portanto, realizar uma revisão ampliada sobre o tema e identificar o "estado da arte" da literatura e suas lacunas (CORDEIRO et al., 2007).

Sendo assim, realizou-se busca no PubMed no dia 27 de maio de 2016, com a seguinte chave de busca: (drug[TitlelAbstract] AND shortage[Title/Abstract]). Os resultados foram exportados para o software de gerenciamento bibliográfico Zotero. O PubMed foi escolhido por ser a principal base de dados da área da saúde.

A bibliografia exportada para o software foi inicialmente selecionada pela leitura do título e resumo com o intuito de retirar os itens que não versavam sobre o tema do desabastecimento. Após essa seleção, foram buscadas, via Periódicos Capes, o 
texto completo dos artigos selecionados. Os itens para os quais não houve acesso ao texto completo foram excluídos desta revisão.

Os textos completos recuperados foram lidos e extraídas em planilha do Libreoffice as seguintes informaçôes: ano de publicação, objetivo e país de realização do estudo, método, medicamento(s) citado(s) como casos de desabastecimento, principais resultados e conclusóes. Quando o artigo era editorial, carta ao editor, comentário, ou seja, não era um estudo com o uso de dados empíricos, esta caracterização foi registrada no campo de "método". Ainda, ao longo da fase de seleção, foi possível perceber que se podia classificar os artigos segundo seu foco principal, permitindo relacioná-los às seguintes categorias: consequência, causa do desabastecimento, estratégias de enfrentamento frente a esta situação ou discussão conceitual do problema. Embora um mesmo artigo possa abranger mais de uma dessas dimensões, eles foram classificados em apenas uma dimensão, de acordo com seu foco principal. Tal decisão foi tomada baseada, principalmente, no título e no objetivo do estudo.

As referências retidas foram descritas de maneira quantitativa obtendo frequências de acordo com o ano de publicação, país de realização, produtos para a saúde citados e foco principal. Ademais, os medicamentos citados nos estudos retidos foram categorizados até o nível 2 da classificação ATC (Anatomical Therapeutic Chemical) da OMS (WHO COLLABORATING CENTRE FOR DRUG STATISTICS METHODOLOGY, 2012). Foi realizada, também, uma descrição dos estudos incluídos, buscando relatar seus principais resultados e conclusôes.

\section{Resultados}

A busca resultou em 791 itens, dos quais foram selecionados 151 após a leitura do título e, caso fosse necessário, resumo dos mesmos. Após a busca pelo texto completo, restaram 98 itens. A árvore da busca e seleção com o detalhamento dessas etapas está ilustrada pela figura 1 . 
Figura 1. Árvore de busca

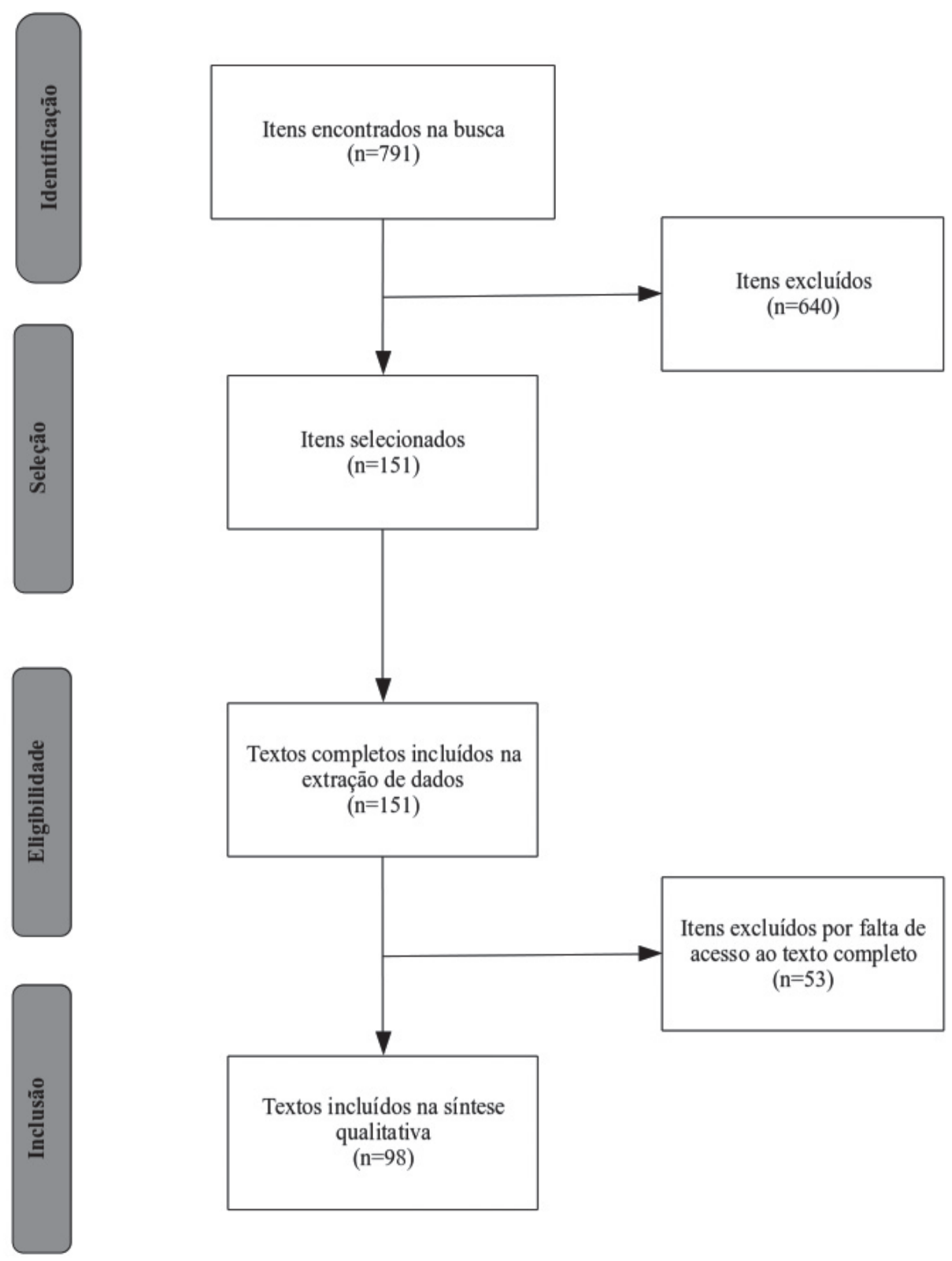

Fonte: elaboração própria. 
O gráfico 1 apresenta os artigos por ano de publicação. Percebe-se que o número de publicaçóes neste campo é recente, com a grande maioria concentrada nos últimos seis anos (2011-2015). É importante ressaltar que o dado referente a 2016 não representa a totalidade dos artigos publicados nesse ano, e sim aqueles indexados na base de dados até a data da busca (27/05/2016).

\section{Gráfico 1. Número de itens por ano de publicação}

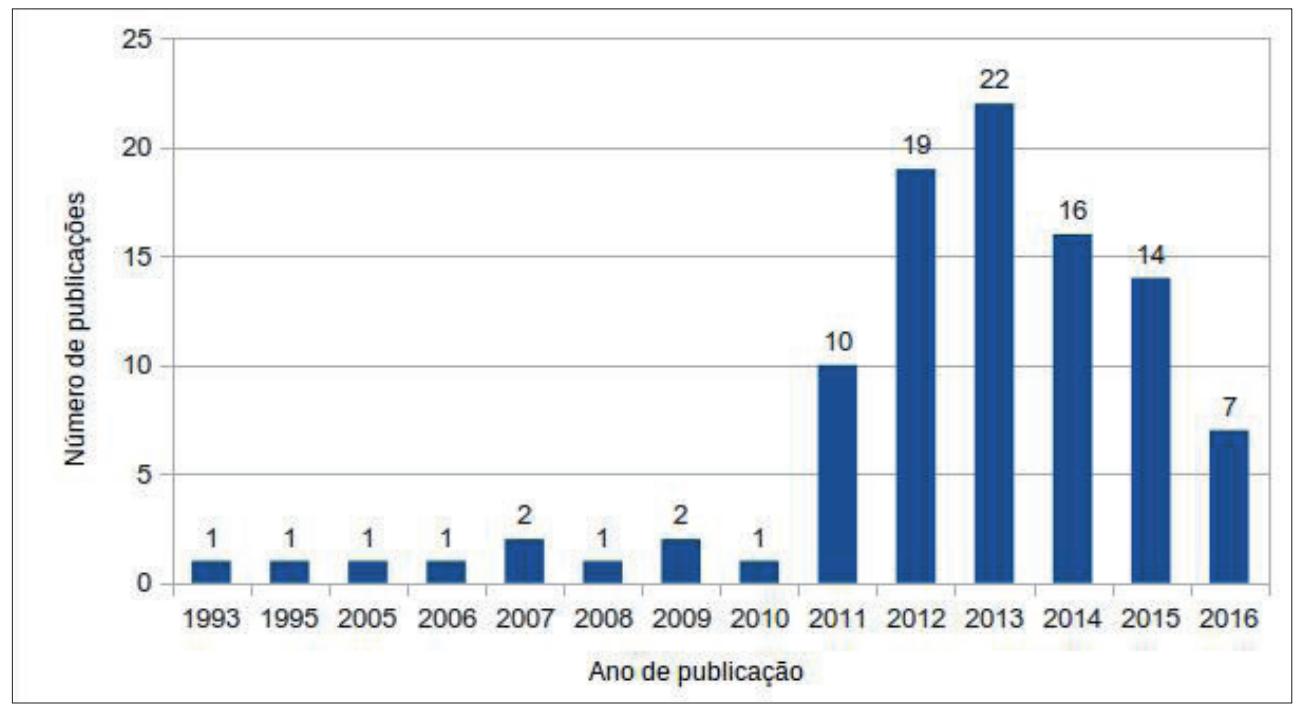

Em relação ao país no qual o estudo foi realizado, a maioria está concentrada nos EUA e, principalmente, nos países de alta renda (gráfico 2). 
Gráfico 2. Número de itens por país foco da publicação

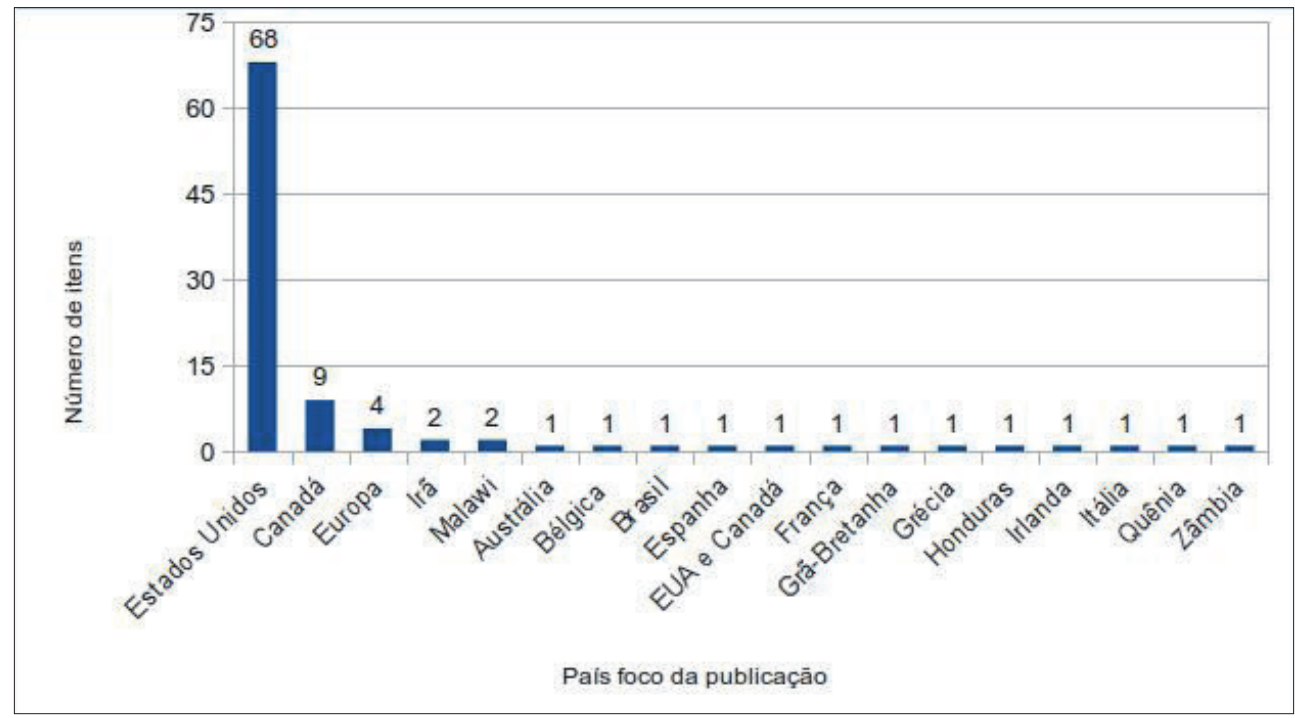

A tabela 1 demonstra os produtos para a saúde citados nas publicaçóes retidas. Há uma clara predominância de artigos que abordam diversos medicamentos, sem focar em uma classe ou medicamento específico. Considerando o código ATC, a classe mais citada (L) refere-se a agentes antineoplásicos e imunomoduladores, sendo o antineoplásico (L01) o mais citado dentro da classe. Aproximadamente 12\% dos medicamentos citados referem-se a substâncias que atuam no sangue e órgãos hematopoiéticos (B) e sistema nervoso $(\mathrm{N})$. Dentro da classe $\mathrm{B}$, os agentes que atuam como substitutos do sangue e soluçôes de perfusão (B05) são os principais medicamentos citados nas referências, principalmente quanto à nutrição parenteral e seus componentes. $\mathrm{Na}$ classe $\mathrm{N}$, a distribuição não é tâo concentrada em uma subclasse como na B; no entanto, é possível observar uma prevalência dos anestésicos (N01) como os medicamentos mais citados. Vale ressaltar o grande número de citaçôes relacionadas a medicamentos oncológicos para a população pediátrica e injetáveis em geral. 
Tabela 1. Frequência simples dos medicamentos citados nas publicaçóes incluídas na revisão segundo seu código ATC/OMS

\begin{tabular}{|c|c|c|c|}
\hline $\begin{array}{c}\text { Código } \\
\text { ATC }\end{array}$ & Descrição & Número & Percentual \\
\hline A & Trato alimentar e metabolismo & 4 & $4,08 \%$ \\
\hline A02 & Medicamentos para transtornos relacionados à acidez & 1 & $1,02 \%$ \\
\hline A11 & Vitaminas & 1 & $1,02 \%$ \\
\hline A16 & Outros produtos do trato alimentar e metabolismo & 2 & $2,04 \%$ \\
\hline $\mathrm{B}$ & Sangue e órgãos hematopoiéticos & 12 & $12,24 \%$ \\
\hline B03 & Preparaçóes antianêmicas & 1 & $1,02 \%$ \\
\hline B05 & Substitutos sanguíneos e soluções de perfusão & 11 & $11,22 \%$ \\
\hline $\mathrm{C}$ & Sistema Cardiovascular & 1 & $1,02 \%$ \\
\hline $\mathrm{C} 08$ & Bloqueadores de canais de cálcio & 1 & $1,02 \%$ \\
\hline $\mathrm{D}$ & Dermatológicos & 2 & $2,04 \%$ \\
\hline D08 & Antissépticos e desinfetantes & 2 & $2,04 \%$ \\
\hline $\mathrm{J}$ & Anti-infecciosos gerais para uso sistémico & 7 & $7,14 \%$ \\
\hline J01 & Antibacterianos de uso sistêmico & 4 & $4,08 \%$ \\
\hline J04 & Antimicobacterianos & 2 & $2,04 \%$ \\
\hline J07 & Vacinas & 1 & $1,02 \%$ \\
\hline $\mathrm{L}$ & Agentes antineoplásicos e imunomoduladores & 14 & $14,29 \%$ \\
\hline L01 & Agentes antineoplásicos & 11 & $11,22 \%$ \\
\hline L03 & Imunoestimulantes & 1 & $1,02 \%$ \\
\hline L04 & Imunossupressores & 2 & $2,04 \%$ \\
\hline $\mathrm{N}$ & Sistema Nervoso & 12 & $12,24 \%$ \\
\hline N01 & Anestésicos & 6 & $6,12 \%$ \\
\hline N03 & Anticonvulsivantes & 1 & $1,02 \%$ \\
\hline N04 & Antiparkinsonianos & 1 & $1,02 \%$ \\
\hline N05 & Psicolépticos & 2 & $2,04 \%$ \\
\hline N07 & Outros medicamentos para o sistema nervoso & 2 & $2,04 \%$ \\
\hline $\mathrm{P}$ & Produtos antiparasitários, insecticidas e repelentes & 4 & $4,08 \%$ \\
\hline P01 & Antiprotozoários & 2 & $2,04 \%$ \\
\hline
\end{tabular}




\begin{tabular}{r|l|c|c}
\hline \multicolumn{1}{c|}{$\begin{array}{c}\text { Código } \\
\text { ATC }\end{array}$} & \multicolumn{1}{c|}{ Descriçáo } & Número & Percentual \\
\hline P02 & Anti-helmínticos & 1 & $1,02 \%$ \\
\hline P03 & $\begin{array}{l}\text { Ectoparasitários, incluindo escabicidas, inseticidas e } \\
\text { repelentes }\end{array}$ & 1 & $1,02 \%$ \\
\hline V & Vários & 2 & $2,04 \%$ \\
\hline V04 & Agentes diagnósticos & 2 & $2,04 \%$ \\
\hline Não especificado (variados)* & 26 & $26,53 \%$ \\
\hline Sem classificação** & 11 & $11,22 \%$ \\
\hline Não se aplica*** & 3 & $3,06 \%$ \\
\hline Total & $\mathbf{9 8}$ & - \\
\hline
\end{tabular}

Legenda: *Foram incluídos nesta categoria todos aqueles estudos que abordam o desabastecimento de maneira generalista, sem especificar um medicamento ou classe farmacológica específica. ${ }^{* *}$ Estáo nesta categoria todos os medicamentos para os quais não foi encontrado um código ATC. ${ }^{* * *}$ Não se aplica aos estudos nos quais o foco náo foi o medicamento.

Fonte: elaborado pelos autores.

Observam-se as diferenças nas abordagens adotadas. Há uma predominância de artigos que apresentam a descrição do desabastecimento, suas consequências e estratégias de enfrentamento; entretanto, são poucos os artigos que discutem as causas do desabastecimento ou até mesmo façam uma discussão conceitual do tema (Tabela 2). Importante ressaltar, também, o grande número de publicaçôes (27) que são notas, cartas ao editor, comentários, artigos de opinião, comunicação, entre outros.

Tabela 2. Número de publicaçôes por abordagem

\begin{tabular}{l|l}
\hline \multicolumn{1}{c|}{ Abordagem do estudo } & \multicolumn{1}{c}{ Frequência simples } \\
\hline Descritivo & 40 \\
\hline Consequência & 26 \\
\hline Estratégia de enfrentamento & 26 \\
\hline Causas & 3 \\
\hline Conceitual & 3 \\
\hline Total & $\mathbf{9 8}$ \\
\hline
\end{tabular}

Fonte: elaborado pelos autores. 
Das 40 publicaçôes que focaram na descrição do desabastecimento, metade foi composta de comunicações, editoriais, artigos de opinião, entre outras, que, em geral, clamam por ações para sua solução, comunicam o desabastecimento ou propóem alternativas terapêuticas para substituição ao item desabastecido (ALSPACH, 2012b; BUTLER, 2013; CARTER, 2011; CDC, 2013; DAL MORO, 2013; ELZAWAWY; KERR, 2013; FDA, 1993; FOX; TYLER, 2013; GOMELLA, 2012; KAPOSY, 2014; MAYER, 2012; MIRTALLO, 2011; MORRISON, 1995; NAVARRO et al., 2012; PRINTZ, 2012; TOMLIN, 2016; TRAYNOR, 2011; TURNER et al., 2013; WENZEL, 2015). As demais ressaltam a gravidade do problema, demonstrando que o mesmo é crescente ao longo dos últimos anos e já faz parte do cotidiano dos profissionais de saúde, e relatam casos em unidades de saúde e seus impactos na saúde dos pacientes (ALSPACH, 2012a; BAUTERS et al., 2015; CHOMBA et al., 2010; COSTELLOE et al., 2015; GHOLAMI et al., 2012; GUNDLAPALLI et al., 2013; HAWLEY et al., 2016; HVISDAS et al., 2013; KAFANTARIS, 2012; KANGWANA et al., 2009; KWEDER; DILL, 2013; MAZER-AMIRSHAHI et al., 2014; McKEEVER; BLOCH; BRATIC, 2013; MULLINS; COOK, 2011; PALM; DOTSON, 2015; PAUWELS et al., 2014, 2015; QUADRI et al., 2015; REED et al., 2016; VENTOLA, 2011).

As publicaçóes com foco nas consequências do desabastecimento, diferentemente das pertencentes às demais categorias, são em sua maioria estudos empíricos, com apenas uma exceção (RUKTANONCHAI et al., 2014). ${ }^{1}$ São citadas como consequências: a substituição para terapias menos eficazes e mais tóxicas aos pacientes (BECKER et al., 2013; KEHL et al., 2015; KOSAREK et al., 2011); maiores custos (BIBLE et al., 2014; DORSEY et al., 2009; HAVRILESKY et al., 2012; HAYES et al., 2014; McLAUGHLIN et al., 2013; RALLS et al., 2012); piora de parâmetros bioquímicos (CORRIGAN; KIRBY, 2012; DAVIS; JAVID; HORSLEN, 2014; PRAMYOTHIN et al., 2013); atraso em ensaios clínicos (SALAZAR et al., 2015) e aumento na taxa de eventos adversos (HALL et al., 2013; HOLCOMBE, 2012; McLAUGHLIN et al., 2013; SHETH et al., 2005; WUERZ; BOW; SEFTEL, 2013); e piores desfechos clínicos devido à suspensão da terapia (RUKTANONCHAI et al., 2014). Em contraste com esses resultados, oito artigos não encontraram diferenças importantes ou, inclusive, observaram melhores desfechos clínicos de pacientes na suspensão do tratamento ou redução da dose da terapia (DEROMA et al., 2012; GOLDBLATT et al., 2011; HUGHES; GOSWAMI; MORRIS, 2015; 
ROBERTS et al., 2012; STOREY et al., 2016; THOMA et al., 2014; TOLIA et al., 2014). Um achado interessante foi o de Liang e Mackey (2012), que encontraram os medicamentos que estavam em falta sendo vendidos na internet a preços exorbitantes, sustentando a afirmação de que o desabastecimento fomenta um mercado paralelo de vendas desses medicamentos.

As publicações que trataram das estratégias de enfrentamento relataram casos nos quais as mesmas foram empregadas; apresentaram também consenso de especialistas sobre o manejo do desabastecimento, mostraram as percepçôes dos profissionais quanto às ações necessárias ou, nos casos dos editorais e comentários, advogaram por determinadas açóes para o manejo do desabastecimento (BARLAS, 2013; 2014; CDC, 2007; EGGERTSON, 2011; SIRRS, 2011; VOGEL, 2012). As principais estratégias de enfrentamento citadas são incentivos fiscais e sanitários às empresas em troca de produção continuada e alteração do sistema de fiscalização (SCHWEITZER, 2013); garantia de informação para prescritores, pacientes e farmácia sobre o desabastecimento (HSIA et al., 2015; MIRTALLO et al., 2012); garantia de um estoque de emergência de medicamentos críticos (PETER, 2006); elaboração de um plano de ação para casos de desabastecimento, elencando prioridades de atendimento, estratégias de gestão e possíveis alternativas terapêuticas (BECK et al., 2015; DECAMP et al., 2014; KRISL; FORTIER; TABER, 2013; PLOGSTED et al., 2016a, 2016b; SINGLETON et al., 2013; VALGUS et al., 2013); uso de alternativas terapêticas, mesmo que sejam off-label (BERTHE-AUCEJO et al., 2014; LEMOS; WAIGNEIN; HAAN, 2016); estabelecimento de parceria com as empresas (JENSEN; THROCKMORTON, 2015); contratação de farmacêuticos especificamente para monitorar os estoques e manejar situaçôes de desabastecimento (CAULDER et al., 2015); treinamento de profissionais de saúde (EGGERTSON, 2011; JAGSI et al., 2014); discussão sobre o uso de medicamentos vencidos em casos críticos (LEMOS et al., 2012); uso de sistemas de informação para monitoramento dos eventos adversos decorrentes do desabastecimento (McLAUGHLIN et al., 2014); e uso de técnicas alternativas de infusão para redução da dose necessária (McHUGH; IBINSON, 2013; PARBHOO et al., 2014).

Dos três artigos que focaram nas causas do fenômeno, dois (LUFESI; ANDREW; AURSNES, 2007b; WOODCOCK; WOSINSKA, 2012) eram estudos empíricos, enquanto um era um editorial que enfatizava a importância de sua investigação (BLUM, 2014). Dos estudos empíricos, Lufesi et al (2007b) identificam como 
causa do desabastecimento de medicamentos no Malawi a deficiência do sistema de distribuição local. O estudo de Woodcock e Wosinka (2012), ao analisarem o desabastecimento de injetáveis nos Estados Unidos, aponta como causa problemas relacionados à qualidade dos medicamentos que levam à interrupção de sua produção. Por meio de uma revisão bibliográfica, os autores apontam que a produção de injetáveis é mais complexa e mais cara e, adicionalmente, sua qualidade não pode ser aferida pelos consumidores finais. Assim, os produtores investem pouco em infraestrutura, ocasionando, eventualmente, problemas na fabricação que podem ter como consequência o desabastecimento. Este problema se agrava tendo em vista o pequeno número de produtores de injetáveis. Portanto, para eles, a fim de evitar futuros desabastecimentos, seria importante implantar um sistema que recompensasse os produtores pela qualidade de seus medicamentos, funcionando como um incentivo financeiro para a produção de injetáveis de maior qualidade, e tendo como consequência menos problemas de interrupção de fabricação e sua maior disponibilidade (WOODCOCK; WOSINSKA, 2012).

Em relação aos artigos que se detêm a uma discussão conceitual, um é um artigo de opinião (DUFFY, 2012) e dois são trabalhos empíricos (DE WEERDT et al., 2015; REIS; PERINI, 2008). O trabalho de De Weerdt et al. (2015) busca, mediante revisão da literatura científica e cinzenta e entrevistas, definir o conceito de "drug shortage" (desabastecimento, em português) para a comunidade europeia. Para tanto, foram feitas buscas bibliográficas em duas bases e busca manual em sites de órgãos regulatórios e de representação de categorias profissionais. Os autores investigaram, ainda, qual seria o período de tempo no qual poderia se considerar que o produto esteja em desabastecimento. Como conclusôes apontam que, na atualidade, há duas definições gerais de desabastecimento, uma referente a quando notificar as agências reguladoras e outra para a sua designação; assim, falta uma definição que articule essas duas perspectivas a fim de facilitar a comparação entre os diferentes sistemas de informação e estudos científicos. Já Reis e Perini (2008), autores do único estudo brasileiro sobre o tema, baseado em uma revisão de literatura, fazem uma discussão abrangente sobre a questão do desabastecimento, apontando possíveis causas, consequências para a assistência à saúde e analisando potenciais estratégias para seu enfrentamento. Por ser a única publicação encontrada na base de dados que considera o contexto brasileiro, o mesmo torna-se pioneiro na discussão do tema em nível nacional. 


\section{Discussão}

Os resultados demonstram a preponderância de estudos sobre o desabastecimento realizados nos EUA, focados na descrição do problema, publicados entre os anos 2011-2015 e que citam medicamentos variados, nutrição parenteral e seus componentes ou oncológicos. Ressalta-se o grande número de publicaçóes que denunciam o desabastecimento de medicamentos através de editoriais, cartas ao editor etc. Tal revisão demonstrou que este é um problema emergente mas que apresenta importantes lacunas sobre o conhecimento de suas causas e conceito. Adicionalmente, são escassos os estudos que abordam o desabastecimento de medicamentos em países de baixa e média renda.

É importante ressaltar a grande quantidade de publicaçôes tais como cartas, editoriais etc, sugerindo a utilização das revistas científicas como veículos para a denúncia do problema e, talvez, uma estratégia para sensibilizar pesquisadores, docentes e profissionais e, consequentemente, tornar visível um problema invisível que se manifesta no contexto dos serviços de saúde e podem estar sendo ignorados pelos trabalhos acadêmicos.

O grande número de estudos realizados nos EUA pode significar que o desabastecimento é um problema de grande impacto naquele país. No entanto, o mais provável é que este seja apenas o reflexo da tendência mundial de publicaçóes, no qual os EUA se mantêm na primeira colocação (NATURE, 2016). A hipótese de que o desabastecimento de medicamentos seja um problema localizado não se sustenta pelas recentes discussóes sobre o tema em arenas internacionais que o ratificam como um problema mundial (WORLD HEALTH ORGANIZATION, 2015, 2016a, 2017, 2018) e nos relatos dos estudos que descrevem as causas do desabastecimento, derivados de problemas na produçáo dos medicamentos de um único produtor, sendo muitas dessas fábricas localizadas em outros países (LUFESI; ANDREW; AURSNES, 2007a; WOODCOCK; WOSINSKA, 2013). Um dos estudos retidos nesta revisão questiona a falta de publicaçóes neste tema provenientes da Europa (PAUWELS et al., 2014), onde diversas ações regulatórias já foram implementadas.

A grande quantidade de artigos abordando o desabastecimento de constituintes da nutrição parenteral diverge do estudo do Institute for Healthcare Informatics (IMS, atualmente IQVIA) realizado nos EUA, que direcionava a problemática 
para oncológicos, antimicrobianos, cardiovasculares, medicamentos do sistema nervoso central e de controle da dor (IMS, 2011). De qualquer maneira, chama a atenção o cenário, uma vez que os medicamentos mais citados são os utilizados em situações críticas, como os anestésicos, oncológicos, nutrição parenteral e seus componentes e injetáveis. Esses medicamentos em si têm pouca possibilidade de substituição, colocando os pacientes em situação de maior vulnerabilidade. Ressaltase que a população pediátrica já tem um rol mais restrito de opçôes (IVANOVSKA; MANTEL-TEEUWISSE; DIJK, 2013), e também foi descrito como alvo de situações de desabastecimento.

Aprofundar o conhecimento sobre esse problema torna-se passo fundamental para a discussão de como resolvê-lo. Uma hipótese a ser investigada é se produtos antigos e clinicamente efetivos estâo sendo retirados do mercado pelos fabricantes e sendo substituídos por outros mais novos e, muitas vezes, em situação de monopólio.

A concentração de publicaçôes nos últimos anos sugere a contemporaneidade do problema. O aumento das publicaçóes em 2011 coincide com a assinatura do ato executivo do presidente Barack Obama que permite que a agência regulatória americana requeira notificação de desabastecimento e faça uma revisão sanitária de instalaçôes que poderiam produzir os medicamentos em falta (GEHRETT, 2012). É possível fazer essa relação, uma vez que a maioria das publicaçóes retidas é dos EUA. Este fato demonstra que 2011 foi o ano no qual o desabastecimento emergiu como um problema relevante naquele país.

Em termos regulatórios, inclusive nos artigos que abordam as estratégias de enfrentamento, é importante notar que, em sua maioria, os textos são reativos e dirigem-se ou a aumentar a informação sobre o desabastecimento ou a estratégias de gerenciamento farmacêutico (troca de medicamentos, estoque, reserva etc.). Por exemplo, no Brasil, a exemplo da regulação europeia, a resposta regulatória foi a exigência da notificação de interrupção de produção e/ou importação de medicamentos (BRASIL, 2014b) e a criação, em 2015, de um grupo de trabalho para "elaborar diagnóstico situacional dos medicamentos no país com risco de redução da oferta, e propor estratégias para mitigação e gestão dos riscos no âmbito do SUS” (BRASIL, 2015). No caso da L-asparaginase, quando a única empresa detentora do registro interrompeu a distribuição do medicamento no país, a resposta foi importar de fornecedores estrangeiros por cotação de menor preço (JORNAL DO BRASIL, 2017). 
É interessante notar que, na maioria dos artigos revisados, há uma suposta responsabilização dos gestores pela falta de treinamento, programação e alternativas que visem evitar o desabastecimento. Porém, mesmo que haja planejamento interno que tente minimizar as causas de uma provável escassez de medicamentos, quando o desabastecimento ocorre, seja no mercado nacional ou internacional, nada pode ser feito pelo gestor local. Nesse sentido, é preocupante o grande número observado de referências que enfocam as estratégias de enfrentamento na regulação da demanda e garantia de disponibilidade em reação à reduzida oferta (ou, em alguns casos, nenhuma). Pois esse foco negligencia a necessidade de se pensar em estratégias que também se voltem para a garantia da oferta dos medicamentos no mercado, sendo esta, portanto, uma lacuna importante neste tema.

De certa forma, esta visão preponderante da demanda no tema de desabastecimento também se reflete nos achados desta revisão, no qual foi possível detectar a escassez de estudos que se voltem a pesquisar as causas deste fenômeno, sendo esta outra importante lacuna do tema. Assim, faltam estudos que ampliem o olhar, deslocandose das questôes logísticas e buscando, também, explicaçôes na organização do setor farmacêutico, em como as decisôes de produção são tomadas neste setor, em determinantes do setor regulatório, em sua estrutura de pesquisa e desenvolvimento, em suas relações com o sistema financeiro, e na própria distribuição de renda e carga de doenças pelo mundo.

Uma terceira lacuna encontrada é relativa à definição de "desabastecimento" de medicamentos. Este termo ainda carece de uma definição que permita o diálogo entre as publicaçóes e, inclusive, de açóes regulatórias mais precisas para seu enfrentamento (DE WEERDT et al., 2015). Adicionalmente, é importante ter pesquisas que subsidiem a discussão desse problema em todos os âmbitos e em todos os países. No Brasil, é sabido que há problema de desabastecimento de medicamentos e relatórios do Ministério da Saúde (BRASIL, 2016; 2014; 2015) inclusive confirmam essa situação; no entanto, não há pesquisas que abordem a questão em âmbito nacional.

Por fim, é importante discutir as limitaçôes deste estudo. Apesar de não ter utilizado critérios de seleção restritivos, este estudo usou apenas uma fonte de busca, o PubMed. Embora esta seja a principal base de dados da área da saúde, é possível que haja publicaçôes do assunto em outras áreas e de outros países que não foram discutidas aqui. Ademais, a chave de busca, por ser muito específica, pode ter introduzido um viés de seleção, muito embora o termo "drug shortage" seja 
conhecido e muito utilizado para o problema do desabastecimento de medicamentos. Assim, os resultados aqui descritos referem-se a publicaçóes desta base nas quais houve acesso ao texto completo (aberto ou disponível via Portal Capes). Ainda assim, esta pesquisa traz uma grande contribuição ao apontar lacunas nos estudos de desabastecimento de medicamentos.

\section{Conclusão}

O desabastecimento de medicamentos é um problema de saúde pública que vem atingindo os sistemas e afeta diretamente o cuidado em saúde, tendo consequências importantes para a qualidade de vida das populaçóes. O presente estudo constatou que esse problema está afetando medicamentos usados para doenças prevalentes que afetam países de alta renda e que geralmente têm altos preços de mercado. Apesar de sua importância e impacto bem documentado nos cuidados de saúde, ainda existem lacunas importantes no estudo do desabastecimento de medicamentos. Especialmente importante é a pesquisa adicional que investigue as causas desse problema; que faça discussóes conceituais para encontrar uma linguagem comum a fim de permitir a comparação entre estudos e estratégias regulatórias; pesquisas em países de baixa e média renda; e estudos que ampliem a perspectiva sobre o problema não se concentrando apenas no ciclo logístico dos serviços farmacêuticos e nas estratégias de enfrentamento das unidades de saúde. Ademais, é importante aprofundar a análise sobre a estratégia de direcionamento da produção de medicamentos para produtos de maior preço unitário no desabastecimento de medicamentos.

Lidar adequadamente com o desabastecimento de medicamentos provavelmente necessitará de estratégias de enfrentamento mais abrangentes. No entanto, tais estratégias só serão efetivas na resolução do problema se o conhecermos bem, se tivermos evidências robustas de suas causas e de como isso afeta diferentes lugares no mundo. ${ }^{2}$

\section{Referências}

BRASIL. Ministério da Saúde. Agência Nacional de Vigilância Sanitária. Resolução da Diretoria Colegiada-RDC no 18, de 4 de abril de 2014. Rio de Janeiro: ANS, 2014b, p. 3.

ALSPACH, J. G. The drug shortage: what Critical Care Nurse readers report. Critical Care Nurse, v. 32, n. 5, p. 11-13, 10 jan. 2012a. 
ALSPACH, J. G. Is the drug shortage affecting patient care in your critical care unit? Critical Care Nurse, v. 32, n. 1, p. 8-13, fev. 2012b.

BARLAS, S. FDA Strategies to prevent and respond to drug shortages. Pharmacy and Therapeutics, v. 38, n. 5, p. 261-263, maio 2013.

BARLAS, S. Manufacturers and hospitals spar over drug shortage reporting. Pharmacy and Therapeutics, v. 39, n. 3, p. 152-215, mar. 2014.

BAUTERS, T. et al. Chemotherapy drug shortages in paediatric oncology: A 14-year single-centre experience in Belgium. Journal of Oncology Pharmacy Practice, p. 1078155215610915, 6 out. 2015.

BECK, J. C. et al. An ethical framework for responding to drug shortages in pediatric oncology: Ethical Framework for Drug Shortages. Pediatric Blood \& Cancer, v. 62, n. 6, p. 931-934, jun. 2015.

BECKER, D. J. et al. Impact of oncology drug shortages on patient therapy: unplanned treatment changes. Journal of Oncology Practice, v. 9, n. 4, p. e122-128, jul. 2013.

BERTHE-AUCEJO, A. et al. Traitement de la gale et rupture d'Ascabiol ${ }^{\bullet}$ : quid de la population pédiatrique ? Gale chez l'enfant et rupture d'Ascabiol ${ }^{\circledR}$. Archives de Pédiatrie, v. 21, n. 6, p. 670675, jun. 2014.

BIBLE, J. R. et al. Impact of drug shortages on patients receiving parenteral nutrition after laparotomy. Journal of Parenteral and Enteral Nutrition, v. 38, n. 2, supl., p. 65S-71S, nov. 2014.

BLUM, F. C. "Doctor, we have no saline today": the curious case of the generic injectable drug shortage. Academic Emergency Medicine, v. 21, n. 6, p. 699-700, jun. 2014.

BOM DIA BRASIL. Falta penicilina no Brasil para tratar crianças que nascem com sífilis. Bom Dia Brasil, 22 jul. 2016.

BRASIL. Ministério da Saúde. Portaria n 1.626, de 30 de setembro de 2015. Institui Grupo de Trabalho com finalidade de elaborar diagnóstico situacional dos medicamentos no país com risco de redução da oferta, e propor estratégias para mitigação e gestão dos riscos no âmbito do SUS. Brasília: MS, 2015.

BRASIL. Ministério da Saúde. Agência Nacional de Vigilância Sanitária. Resolução da Diretoria Colegiada-RDC no 18, de 4 de abril de 2014. Dispóe sobre a comunicação à Agência Nacional de Vigilância Sanitária - ANVISA dos casos de descontinuação temporária e definitiva de fabricação ou importação de medicamentos, reativação de fabricação ou importação de medicamentos, e dá outras providências. Brasília: MS, $2014 \mathrm{a}$.

BRASIL. Ministério da Saúde. Secretaria de Vigilância em Saúde. Departamento de DST, aids e hepatites virais. Boletim Epidemiológico-Sifilis. [s.l: s.n.]. Disponível em: <http://www.aids. gov.br/sites/default/files/anexos/publicacao/2015/57978/_p_boletim_sifilis_2015_fechado_ pdf_p__18327.pdf>. Acesso em: 23 set. 2016. 
BUTLER, D. Iran hit by drug shortage. Nature, v. 504, n. 7478, p. 15-16, 5 dez. 2013.

CARTER, D. Drug shortage crisis affects patients and nurses. The American Journal of Nursing, v. 111, n. 11, p. 14, nov. 2011.

CAULDER, C. R. et al. Impact of Drug Shortages on Health System Pharmacies in the Southeastern United States. Hospital Pharmacy, v. 50, n. 4, p. 279-286, abr. 2015.

CENTERS FOR DISEASE CONTROL AND PREVENTION. Deportation of tuberculosis patients complicated by a medication shortage - Honduras, May-August 2006. Morbidity and Mortality Weekly Report, v. 56, n. 26, p. 655-658, 6 jul. 2007.

CENTERS FOR DISEASE CONTROL AND PREVENTION. National shortage of purified-protein derivative tuberculin products. Morbidity and mortality weekly report, v. 62, n. 16, p. 312, 26 abr. 2013.

CHOMBA, E. N. et al. The current availability of antiepileptic drugs in Zambia: implications for the ILAE/WHO "Out of the shadows" campaign. The American Journal of Tropical Medicine and Hygiene, v. 83, n. 3, p. 571-574, set. 2010.

CORDEIRO, A. M. et al. Systematic review: a narrative review. Revista do Colégio Brasileiro de Cirurgióes, v. 34, n. 6, p. 428-431, dez. 2007.

CORRIGAN, M.; KIRBY, D. F. Impact of a national shortage of sterile ethanol on a home parenteral nutrition practice: a case series. Journal of Parenteral and Enteral Nutrition, v. 36, n. 4, p. 476-480, jul. 2012.

COSTELLOE, E. M. et al. An audit of drug shortages in a community pharmacy practice. Irish Journal of Medical Science (1971 -), v. 184, n. 2, p. 435-440, jun. 2015.

DAL MORO, F. BCG shortage in Europe. Preventive Medicine, v. 57, n. 2, p. 146, ago. 2013.

DAVIS, C.; JAVID, P. J.; HORSLEN, S. Selenium deficiency in pediatric patients with intestinal failure as a consequence of drug shortage. Journal of Parenteral and Enteral Nutrition, v. 38, n. 1, p. 115-118, jan. 2014.

DE WEERDT, E. et al. Toward a European definition for a drug shortage: a qualitative study. Frontiers in Pharmacology, v. 6, p. 253, 2015.

DECAMP, M. et al. Chemotherapy Drug Shortages in Pediatric Oncology: A Consensus Statement. Pediatrics, v. 133, n. 3, p. e716-e724, mar. 2014.

DEROMA, L. et al. Did the temporary shortage in supply of imiglucerase have clinical consequences? Retrospective observational study on 34 italian Gaucher type I patients. JIMD reports, v. 7, p. 117-122, 2012.

DORSEY, E. R. et al. Selegiline shortage: Causes and costs of a generic drug shortage. Neurology, v. 73, n. 3, p. 213-217, 21 jul. 2009. 
DUFFY, E. Drug Shortage Crisis Resolution. Journal of Pharmacy Practice, v. 25, n. 6, p. 619620, 1 dez. 2012.

EGGERTSON, L. Drug shortage registry under discussion. Canadian Medical Association Journal, v. 183, n. 10, p. E637-E638, 12 jul. 2011.

ELZAWAWY, A. M.; KERR, D. J. Variation in the availability of cancer drug generics in the United States of America. Annals of oncology: official journal of the European Society for Medical Oncology, v. 24, suppl. 5, p. v17-22, set. 2013.

FOOD AND DRUG ADMINISTRATION. FDA clears the way for ending shortage of TB treatment drug. Public Health Reports, Washington, D.C., v. 108, n. 4, p. 527, ago. 1993.

FOX, E. R.; TYLER, L. S. Call to action: finding solutions for the drug shortage crisis in the United States. Clinical Pharmacology and Therapeutics, v. 93, n. 2, p. 145-147, fev. 2013.

FROST, L. J.; REICH, M. Access: how do good health technologies get to poor people in poor countries? Cambridge, Mass: Harvard University Press, 2008.

GEHRETT, B. K. A prescription for drug shortages. JAMA, v. 307, n. 2, p. 153-154, 2012.

GHOLAMI, K. et al. Three Years Evaluation of Drug Shortages from Educational Pharmacies in Tehran. Iranian Journal of Pharmaceutical Research, v. 11, n. 2, p. 565-572, 2012.

GOLDBLATT, J. et al. Enzyme replacement therapy "drug holiday": Results from an unexpected shortage of an orphan drug supply in Australia. Blood Cells, Molecules and Diseases, v. 46, n. 1, p. 107-110, jan. 2011.

GOMELLA, L. G. Can anyone spare a little indigo carmine? The drug shortage crisis. The Canadian Journal of Urology, v. 19, n. 3, p. 6238, jun. 2012.

GUNDLAPALLI, A. V. et al. Perspectives and concerns regarding antimicrobial agent shortages among infectious disease specialists. Diagnostic Microbiology and Infectious Disease, v. 75, n. 3, p. 256-259, mar. 2013.

HALL, R. et al. Drug shortages in Canadian anesthesia: a national survey. Canadian Journal of Anaesthesia, v. 60, n. 6, p. 539-551, jun. 2013.

HAVRILESKY, L. J. et al. Economic impact of paclitaxel shortage in patients with newly diagnosed ovarian cancer. Gynecologic Oncology, v. 125, n. 3, p. 631-634, jun. 2012.

HAWLEY, K. L. et al. Longitudinal trends in U.S. drug shortages for medications used in emergency departments (2001-2014). Academic Emergency Medicine, v. 23, n. 1, p. 63-69, jan. 2016.

HAYES, M. S. et al. Lessons from the leucovorin shortages between 2009 and 2012 in a medicare advantage population: where do we go from here? American Health \& Drug Benefits, v. 7, n. 5, p. 264-270, ago. 2014. 
HOLCOMBE, B. Parenteral nutrition product shortages: impact on safety. Journal of Parenteral and Enteral Nutrition, v. 36, n. 2, suppl, p. 44S-47S, mar. 2012.

HSIA, I. K.-H. et al. Survey of the national drug shortage effect on anesthesia and patient safety: a patient perspective. Anesthesia \& Analgesia, v. 121, n. 2, p. 502-506, ago. 2015.

HUGHES, K. M.; GOSWAMI, E. S.; MORRIS, J. L. Impact of a Drug Shortage on Medication Errors and Clinical Outcomes in the Pediatric Intensive Care Unit. The Journal of Pediatric Pharmacology and Therapeutics, v. 20, n. 6, p. 453-461, 2015.

HVISDAS, C. et al. US Propofol drug shortages: a review of the problem and stakeholder analysis. American Health \& Drug Benefits, v. 6, n. 4, p. 171-175, 2013.

INSTITUTE FOR HEALTHCARE INFORMATICS. Drug shortages: a closer look at products, suppliers and volume volatility. Parsippany, NJ, Nov 2011. Disponível em: < http:// eyeonfda.com/wp-content/uploads/2011/11/IMS-IHI-Drug-Shortages-Report-Final.pdf>

IVANOVSKA, V.; MANTEL-TEEUWISSE, A. K.; DIJK, L. V. Priority medicines for Europe and the world. "A Public Health Approach to Innovation". Geneva, 2013. Disponível em: $<$ http://www.who.int/medicines/areas/priority_medicines/BP7_1Children.pdf?ua=1>. Acesso em: 1 fev. 2017.

JAGSI, R. et al. Ethical considerations for the clinical oncologist in an era of oncology drug shortages. The Oncologist, v. 19, n. 2, p. 186-192, fev. 2014.

JENSEN, V.; THROCKMORTON, D. C. Shortage of peritoneal dialysis solution and the Food and Drug Administration's response. Clinical Journal of the American Society of Nephrology, v. 10, n. 8, p. 1484-1486, 8 jul. 2015.

JORNAL DO BRASIL. ANM se manifesta sobre importação de Asparaginase Chinesa pelo Ministério da Saúde. Jornal do Brasil, 11 abr. 2017.

KAFANTARIS, A. Greek drug shortage brings call for cost-effective approach. Nature Medicine, v. 18, p. 1719, 2012.

KANGWANA, B. B. et al. Malaria drug shortages in Kenya: a major failure to provide access to effective treatment. The American Journal of Tropical Medicine and Hygiene, v. 80, n. 5, p. 737-738, 5 jan. 2009.

KAPOSY, C. Drugs, money, and power: the Canadian drug shortage. Journal of Bioethical Inquiry, v. 11, n. 1, p. 85-89, mar. 2014.

KEHL, K. L. et al. Oncologists' experiences with drug shortages. Journal of Oncology Practice, v. 11, n. 2, p. e154-162, mar. 2015.

KOSAREK, L. et al. Increase in venous complications associated with etomidate use during a propofol shortage: an example of clinically important adverse effects related to drug substitution. The Ochsner Journal, v. 11, n. 2, p. 143-146, 2011. 
KRISL, J. C.; FORTIER, C. R.; TABER, D. J. Disruptions in the Supply of Medications Used in Transplantation: Implications and Management Strategies for the Transplant Clinician: Critical Drug Shortages in Solid Organ Transplant. American Journal of Transplantation, v. 13, n. 1, p. 20-30, jan. 2013.

KWEDER, S. L.; DILL, S. Drug shortages: the cycle of quantity and quality. Clinical Pharmacology \& Therapeutics, v. 93, n. 3, p. 245-251, 1 mar. 2013.

LEMOS, M. de et al. Using expired supply in times of drug shortage. Journal of Oncology Pharmacy Practice, v. 18, n. 2, p. 319-319, 1 jun. 2012.

LEMOS, M. L. de; WAIGNEIN, S.; HAAN, M. DE. Evidence-based practice in times of drug shortage. Journal of Oncology Pharmacy Practice, v. 22, n. 3, p. 566-570, 1 jun. 2016.

LIANG, B. A.; MACKEY, T. K. Online Availability and Safety of Drugs in Shortage: A Descriptive Study of Internet Vendor Characteristics. Journal of Medical Internet Research, v. 14, n. 1, p. e27, 9 fev. 2012.

LUFESI, N. N.; ANDREW, M.; AURSNES, I. Deficient supplies of drugs for life threatening diseases in an African community. BMC health services research, v. 7, p. 86, 2007a.

LUFESI, N. N.; ANDREW, M.; AURSNES, I. Deficient supplies of drugs for life threatening diseases in an African community. BMC Health Services Research, v. 7, p. 86, 15 jun. 2007 b.

MAYER, D. K. Anatomy of a drug shortage. Clinical Journal of Oncology Nursing, v. 16, n. 2, p. 107-108, abr. 2012.

MAZER-AMIRSHAHI, M. et al. Critical Drug Shortages: Implications for Emergency Medicine. Academic Emergency Medicine, v. 21, n. 6, p. 704-711, jun. 2014.

MCHUGH, S. M.; IBINSON, J. W. Anesthesiology in the era of drug shortages: use of a succinylcholine infusion for a laparoscopic sigmoid colectomy due to a shortage of neostigmine. Journal of Clinical Anesthesia, v. 25, n. 1, p. 79-81, fev. 2013.

MCKEEVER, A. E.; BLOCH, J. R.; BRATIC, A. Drug shortages and the burden of access to care: a critical issue affecting patients with cancer. Clinical Journal of Oncology Nursing, v. 17, n. 5, p. 490-495, 1 Oct. 2013.

MCLAUGHLIN, M. et al. Effects on patient care caused by drug shortages: a survey. Journal of Managed Care Pharmacy, v. 19, n. 9, p. 783-788, Dec. 2013.

MCLAUGHLIN, M. M. et al. Developing a Method for Reporting Patient Harm Due to Antimicrobial Shortages. Infectious Diseases and Therapy, v. 3, n. 2, p. 349-355, dez. 2014.

MICHAELIS ON-LINE. Dicionário Brasileiro da Lingua Portuguesa. Disponível em: <http:// michaelis.uol.com.br/busca? $\mathrm{r}=0 \& \mathrm{f}=0 \& \mathrm{t}=0 \&$ palavra=desabastecimento $>$. Acesso em: 30 set. 2016. 
MIRTALLO, J. M. The drug shortage crisis. Journal of Parenteral and Enteral Nutrition, v. 35, n. 4, p. 433, Jul. 2011.

MIRTALLO, J. M. et al. Parenteral nutrition product shortages The A.S.P.E.N. Strategy. Nutrition in Clinical Practice, v. 27, n. 3, p. 385-391, 1 Jun. 2012.

MORRISON, G. Malaria prophylaxis. Generous prescribing advised for travelling to areas of drug shortage. $B M J$, v. 311, n. 6998, p. 191-192, 15 jul. 1995.

MUlLINS, T. D.; COOK, A. M. Drug Shortages: Causes and Cautions. Orthopedics, v. 34, n. 9, p. 712-714, 1 set. 2011.

NATURE. Nature Index 2016 tables. Disponível em: <https://www.natureindex.com/annualtables/2016/country/all>. Acesso em: 1 Feb. 2017.

NAVARRO, M. et al. Benznidazole shortage makes chagas disease a neglected tropical disease in developed countries: data from Spain. The American Journal of Tropical Medicine and Hygiene, v. 87, n. 3, p. 489-490, Sept. 2012.

PALM, E.; DOTSON, B. Copper and zinc deficiency in a patient receiving long-term parenteral nutrition during a shortage of parenteral trace element products. Journal of Parenteral and Enteral Nutrition, v. 39, n. 8, p. 986-989, 1 nov. 2015.

PARBHOO, R. K. et al. Innovative approach to preparing radial artery cocktails in response to manufacturer shortages of nitroglycerin and verapamil. Hospital Pharmacy, v. 49, n. 7, p. 628-633, Jul. 2014.

PAUWELS, K. et al. Drug shortages in European countries: a trade-off between market attractiveness and cost containment? BMC health services research, v. 14, p. 438, 2014.

PAUWELS, K. et al. Insights into European drug shortages: a survey of hospital pharmacists. PLoS ONE, v. 10, n. 3, 16 Mar. 2015.

PETER, G. Tailoring the Strategies to Specific Shortages: Pneumococcal Conjugate Vaccine. Clinical Infectious Diseases, v. 42, n. suppl. 3, p. S138-S140, 3 Jan. 2006.

PLOGSTED, S. et al. Parenteral nutrition electrolyte and mineral product shortage considerations. Nutrition in Clinical Practice, v. 31, n. 1, p. 132-134, 1 Feb. 2016 a.

PLOGSTED, S. et al. Parenteral Nutrition Multivitamin Product Shortage Considerations. Nutrition in Clinical Practice, v. 31, n. 4, p. 556-559, 1 Aug. 2016 b.

PRAMYOTHIN, P. et al. Anemia and leukopenia in a long-term parenteral nutrition patient during a shortage of parenteral trace element products in the United States. Journal of Parenteral and Enteral Nutrition, v. 37, n. 3, p. 425-429, Jun. 2013.

PRINTZ, C. Medication shortages threaten cancer care: the oncology community and the FDA tackle ongoing drug shortage problem. Cancer, v. 118, n. 2, p. 289-291, 15 Jan. 2012. 
QUADRI, F. et al. Antibacterial drug shortages from 2001 to 2013: implications for clinical practice. Clinical Infectious Diseases, v. 60, n. 12, p. 1737-1742, 15 Jun. 2015.

RALLS, M. W. et al. Drug shortage-associated increase in catheter-related blood stream infection in children. Pediatrics, v. 130, n. 5, p. e1369-1373, Nov. 2012.

REED, B. N. et al. The impact of drug shortages on patients with cardiovascular disease: causes, consequences, and a call to action. American Heart Journal, v. 175, p. 130-141, May 2016.

REIS, A. M. M.; PERINI, E. Drug shortage: determinants, consequences and management. Ciência \& Saúde Coletiva, v. 13 Suppl, p. 603-610, abr. 2008.

ROBERTS, R. et al. Impact of a national propofol shortage on duration of mechanical ventilation at an academic medical center*. Critical Care Medicine, v. 40, n. 2, p. 406-411, Feb. 2012.

RUKTANONCHAI, D. et al. Zinc deficiency-associated dermatitis in infants during a nationwide shortage of injectable zinc - Washington, DC, and Houston, Texas, 2012-2013. Morbidity and mortality weekly report, v. 63, n. 2, p. 35-37, 17 Jan. 2014.

SALAZAR, E. G. et al. The impact of chemotherapy shortages on COG and local clinical trials: A report from the Children's Oncology Group: COG Chemotherapy Shortage Study. Pediatric Blood \& Cancer, v. 62, n. 6, p. 940-944, Jun. 2015.

SCHWEITZER, S. O. How the US Food and Drug Administration Can Solve the Prescription Drug Shortage Problem. American Journal of Public Health, v. 103, n. 5, p. e10-e14, May 2013.

SHETH, H. S. et al. Promethazine adverse events after implementation of a medication shortage interchange. The Annals of Pharmacotherapy, v. 39, n. 2, p. 255-261, Feb. 2005.

SINGLETON, R. et al. From framework to the frontline: designing a structure and process for drug supply shortage planning. Healthcare Management Forum, v. 26, n. 1, p. 41-45, Apr. 2013.

SIRRS, S. The Fabrazyme shortage: a call to action for metabolic physicians. Molecular Genetics and Metabolism, v. 102, n. 1, p. 4-5, jan. 2011.

STOREY, M. A. et al. Evaluation of parenteral nutrition errors in an era of drug shortages. Nutrition in Clinical Practice, v. 31, n. 2, p. 211-217, 1 abr. 2016.

MARTINS, T. Falta de matéria-prima compromete tratamento de sífilis congênita no Brasil. Rio de Janeiro, 30 jun. 2016. Disponível em: <https://noticias.uol.com.br/saude/ultimasnoticias/redacao/2016/06/30/falta-de-materia-prima-compromete-tratamento-de-sifiliscongenita-no-brasil.htm>.

THOMA, B. N. et al. Clinical and economic impact of substituting dexmedetomidine for propofol due to a US Drug Shortage: Examination of Coronary Artery Bypass Graft Patients at an Urban Medical Centre. PharmacoEconomics, v. 32, n. 2, p. 149-157, 1 Feb. 2014. 
TOLIA, V. N. et al. The Effect of the National Shortage of Vitamin A on Death or Chronic Lung Disease in Extremely Low-Birth-Weight Infants. JAMA Pediatrics, v. 168, n. 11, p. 1039, 1 Nov. 2014.

TOMLIN, S. Where have all the medicines gone? Archives of Disease in Childhood, 12 Feb. 2016.

TRAYNOR, K. Drug shortage solutions elude stakeholders. American Journal of Health-System Pharmacy, v. 68, n. 22, p. 2106, 2108, 15 nov. 2011.

TURNER, R. E. et al. Extent and Effects of Recurrent Shortages of Purified-Protein Derivative Tuberculin Skin Test Antigen Solutions — United States, 2013. Morbidity and Mortality Weekly Report, v. 62, p. 1014-1015, 13 dez. 2013.

VALGUS, J. et al. Ethical challenges: managing oncology drug shortages. Journal of Oncology Practice, v. 9, n. 2, p. e21-e23, mar. 2013.

VENTOLA, C. L. The drug shortage crisis in the United States: causes, impact, and management strategies. Peer-Reviewed Journal for Formulary Management, v. 36, n. 11, p. 740 757, Nov. 2011.

VOGEL, L. Online drug shortage registry limited in application. Canadian Medical Association Journal, v. 184, n. 3, p. E165-E166, 21 fev. 2012.

WENZEL, R. Drug Shortages and Tipping Points. Headache: The Journal of Head and Face Pain, v. 55, n. 9, p. 1294-1295, out. 2015.

WHO COLLABORATING CENTRE FOR DRUG STATISTICS METHODOLOGY. Guidelines for ATC classification and DDD assignment 2013. Oslo: WHO, 2013.

WOODCOCK, J.; WOSINSKA, M. Economic and Technological Drivers of Generic Sterile Injectable Drug Shortages. Clinical Pharmacology \& Therapeutics, 7 Nov. 2012.

WOODCOCK, J.; WOSINSKA, M. Economic and technological drivers of generic sterile injectable drug shortages. Clinical Pharmacology and Therapeutics, v. 93, n. 2, p. 170176, Feb. 2013. WORLD HEALTH ORGANIZATION. EB138/41: Addressing the global shortages of medicines, and the safety and accessibility of children's medication, 18 dez. 2015. . Acesso em: 23 set. 2016

WORLD HEALTH ORGANIZATION. A69/42: Addressing the global shortages of medicines, and the safety and accessibility of children's medication, 24 mar. 2016a. . Acesso em: 23 set. 2016

WORLD HEALTH ORGANIZATION. EB140/19: Addressing the global shortage of medicines and vaccines, Dec. 2016b. Disponível em: <http://apps.who.int/gb/ebwha/pdf_files/EB140/ B140_19-en.pdf>. Acesso em: 14 dez. 2018

WORLD HEALTH ORGANIZATION. EB140/2017/REC/1, jan. 2017. Disponível em: <http://apps.who.int/gb/ebwha/pdf_files/EB140-REC1/B140_REC1-en.pdf\#page=1>. Acesso em: 14 dez. 2018 
WORLD HEALTH ORGANIZATION. EB142/13: Addressing the global shortage of, and access to, medicines and vaccines. Report by the Director-General, 12 jan. 2018. Disponível em: <http:// apps.who.int/gb/ebwha/pdf_files/EB142/B142_13-en.pdf>

WUERZ, T. C.; BOW, E. J.; SEFTEL, M. D. Potential consequences of essential drug shortages in Canada: Brain abscess due to Nocardia farcinica associated with dapsone prophylaxis for Pneumocystis jirovecii pneumonia. The Canadian Journal of Infectious Diseases \& Medical Microbiology, v. 24, n. 3, p. 159-161, 2013.

\section{Notas}

${ }^{1}$ Centro de Controle de Doenças (CDC) dos Estados Unidos (EUA) sobre eventos adversos na falta de um suplemento de zinco.

${ }^{2}$ L.A. Chaves participou da concepção do estudo, busca, seleção e extração das referências, análise dos resultados, escrita, revisão e aprovação do manuscrito final. G. C. Chaves participou da concepção do estudo, escrita, revisão e aprovação do manuscrito final. M. N. S. Vianna participou da seleçáo e extração das referências, análise dos resultados, escrita, revisão e aprovação do manuscrito final. M. A. participou da concepção do estudo, escrita, revisão e aprovação do manuscrito final. 


\section{Abstract}

\section{Medicines shortage in medical scientific journals: a literature review}

The shortage of medicines is already considered a public health problem that affects several regions worlwide and is a major obstacle to ensure access to medicines and, consequently, the right to health. Thus, in order to better understand this phenomenon, this article sought to identify, describe and characterize the medical scientific publications that deal with the issue of medicines shortages and to identify the gaps on this theme. For that, a narrative review of the medical scientific literature was carried out in the PubMed database. The results were selected according to the title and abstract, and the data were extracted from the full text. In addition to quantitative analysis, a qualitative synthesis of the studies was also performed, identifying the mentioned causes, coping strategies, conceptual discussions and problem description. We analyzed 98 papers, most of them published since the year 2011, in the United States, citing various drugs and with a focus on describing the problem. The results of this study suggest the contemporaneous of the problem, the use of scientific literature as drug shortages denunciation, the lack of studies in low- and middle-income settings, and a gap in studies that investigates the causes of medicines shortages.

> Keywords: pharmaceutical preparations; review; health services accessibility. 